\title{
A Stable Routing Protocol for Mobile Ad Hoc Networks
}

\author{
Jinke Huang ${ }^{1, a}$, Xiaoguang Fan ${ }^{1, b}$ Shuai $\mathrm{Li}^{1, \mathrm{c}}$, and Shaohua Chen ${ }^{1, \mathrm{~d}}$ \\ ${ }^{1}$ Aeronautics and Astronautics Engineering College, Air Force Engineering University \\ a86297609@qq.com, 15829717886@163.com, 'lishuailisuai@163.com, ${ }^{\mathrm{d}}$ chensh10@126.com
}

Keywords: mobile ad hoc networks, stable clustering algorithm, hierarchical routing protocol, performance evaluation

\begin{abstract}
Hierarchical routing is one of the fundamental but challenging topics in mobile ad hoc network, which combine proactive/table-driven with reactive/on-demand routing protocols and take advantages of both. And as an imperative means of hierarchical routing protocol, clustering approach provides an efficient and stable hierarchical structure for mobile ad hoc networks. In this paper, we designed a stable clustering algorithm and a corresponding hierarchical routing protocol for high dynamic mobile ad hoc networks. Each cluster is consisted of a cluster-head, several cluster-gateway nodes, several cluster-guest nodes and other cluster-members. The proposed routing protocol uses proactive protocol in inter-cluster and reactive protocol in intra-cluster communication. Simulation results showed that the clustering algorithm improves its stability by increasing the node number of individual clusters and decreasing node's switch times between clusters. And the proposed routing protocol not only decreases the end-to-end delay to guarantee the validity of data but reduces the communication control expenses to increase the utility of network resource and increases the packet acceptance ratio to verify the validity of the proposed protocol.
\end{abstract}

\section{Introduction}

Wireless, multi-hop, mobile ad hoc networks (MANETs) are composed of a collection of mobile independent nodes. It can communicate with each other without fixed based station or any wireless backbone infrastructure. Hence, its applications range from digital battlefield communications, emergency operation, disaster recovery (fire, flood and earthquake), law enforcement (crowd control, search and rescue) and sensor dust. But mobility presents a challenging topic for protocol design since the protocol must adapt to frequent topology changes of MANETs. So, design a scalable and efficient routing protocol is a challenging process, especially in the high dynamic MANETs ${ }^{[1]}$.

In typical MANETs, existing routing protocols of MANETs can be sorted into three categories according to the mechanism of updating ${ }^{[2]}$. 1) Proactive/table-driven: this method requires nodes broadcast routing information periodically to maintain valid routing table at all times, for which will cost much bandwidth. Hence, this mechanism is not suitable for large dynamic networks. 2) Reactive/on-demand: in this case, nodes in the network do not regularly maintain routing table for all destinations, just only when a node has data packets to send to some given destination, it checks its routing table to determine whether it has an reactive route to the destination. If not, the node must perform a route discovery procedure to acquire a route to the destination. Thus, this method does scale well to large population and high mobility networks. 3) Hybrid: it is a better compromise of the first two approaches, namely, it uses reactive (proactive) routing between nodes within individual clusters and proactive (reactive) between clusters.

Self-stabilization is of particular interest to ad hoc network designer and users and is an issue with critical influence on numbers of nodes, routing packers will demand a large percentage of the limited wireless bandwidth and this is exaggerated and exacerbated by the mobility feature often resulting in a high frequent of failure regarding wireless links. To overcome such barriers to success and address the issues of scalability and maintenance of MANETs is essential, "to build hierarchies among the nodes, such that the network topology can be abstracted. This process is commonly referred to as clustering and a cluster structure divides a network into groups called clusters, each of which consists of one cluster-head and some ordinary nodes. In each cluster, the cluster-head controls the communications in intra-cluster and inter-cluster.” In MANETs, as some nodes join, move and leave 
the cluster structure should be updated, whole if a cluster structure changes frequently and drastically (e.g., a node alternates its role from an ordinary node to a cluster-head or joins in or leaves from a cluster), communication overhead may increases. To reduce unnecessary communication and to keep providing stable service for external application using the cluster structure, stable cluster structure is necessary.

According to the topology of networks, routing protocols can be sorted into flat-based routing and hierarchical-based routing. In a flat-based routing ${ }^{[3]}$, all nodes play an equal role and can establish a route by local operation and information feedback among themselves easily. But to large scale network, the frequent topology detection may invalidate the discovered routes, which would lead to high delay and network spending. This phenomenon can be solved efficiently in hierarchical-based routing network. In a hierarchical-based routing ${ }^{[4]}$, all nodes are divided into different clusters (zones). Each cluster elects a cluster-head according to specific rules. Data exchanging between clusters was relayed by gateway node, which disregarding the details of how the relayed data would be transmitted to the destination. In a word, hierarchical-based routing not only play down the network spending by decreasing the number of nodes which participate in routing maintenance, but also increase the network stability by dividing the network into easy-control subnets.

In hierarchical routing protocols, stable clustering algorithm not only can reduce the routing overhead, but also increase the scalability of the network. Some has been proposed in literatures [5] [7], and most of them is to satisfy the specific demands, such as the Lowest-ID cluster, the highest-connectivity and so on. A clustering algorithm known as Lowest-ID, which elects the lowest ID of nodes as the cluster-head ${ }^{[5]}$. In [6], Chiang et al showed that the Lowest-ID performs better than the highest-connectivity algorithm, which elects the cluster-head based on the node degree [16]. The DDVC (Dynamic Doppler Velocity Clustering) and DLDC (Dynamic Link Duration Clustering) algorithm was proposed in [7], both of them are just applied to pseudo-linear highly dynamic MANETs. The MPBC (Mobility Prediction-Based Clustering) in [9] elects the node, which has a low variance of the relative mobility values with respect to its neighbors, take the cluster-head responsibility. Bazzal et al. selected a node with more neighbors as a cluster-head in order to reduce the number of clusters [10]. Theoleyre et al. considered load balance and stability when selecting the cluster-head [11]. This method elects the node with the optimal degree, small mobility, and sufficient energy as a cluster-head. All of them somewhat ignored the scenario, namely, when a node moves out of its cluster-head' transmission range but still has a link to another cluster-member belonging to any cluster-head, whether the initial phrase of clustering will be reestablished or not. And the conclusion in [12] reveal that frequent cluster changing consumes lots of network resources and highly overlapped clusters decrease the efficiency of hierarchical structure. Thus, a stable clustering algorithm must maintain a more stable and less overlapping structure.

Based on the analysis of above, a Stable Clustering Algorithm (SCA), which takes the mobility factors and node's relative degree into account to realize stable cluster structure, was designed and a corresponding routing protocol (SCA-R) was proposed in Section 2 and Section 3, respectively. The experiments and analysis are given in section 4. Finally, Section 5 concludes the paper.

\section{Stable Clustering Algorithm}

\subsection{Cost Metric}

We assume that there are $N$ mobile nodes roaming in a $R \times R \mathrm{Km}$ square, and each moves according to the RRGM (Reference Region Group Mobility Model). Each node can acquire their position and time information through GPS (Beidou), and can also broadcast them to their neighbors. The $\overrightarrow{n_{i}(t)}$ is the position vector of $i$ at $t$ o'clock, where $i=1,2, \ldots, N$. Thus, the distance between $i$ and $j$ at $t$ o'clock is $d_{i j}(t)=\left|\overrightarrow{n_{i}(t)}-\overrightarrow{n_{j}(t)}\right|$. Thus, the remote-function of node $i$ and $j$ is $R_{i j}(t)=F\left(d_{i j}(t)\right)$. where $F($.$) is a function of distance.$

The most simply $F($.$) is identity function, namely,$

$$
R_{i j}(t)=d_{i j}(t)
$$


The remote-function is changing along with the motivation of a node. Thus, mobility factor should be denoted by the derivation of remote-function, namely:

$$
M_{i}(t)=\frac{1}{N} \sum_{j=1}^{N}\left|\frac{d}{d t} F\left(d_{i j}(t)\right)\right|
$$

Importing equation 1 into (2), $M_{i}(t)$ can simplified into:

where $\left(t_{2}-t_{1}\right) \rightarrow 0$.

$$
\begin{aligned}
M_{i}(t) & =\frac{1}{N} \sum_{j=1}^{N}\left|d_{i j}^{\prime}(t)\right| \\
& =\frac{1}{N} \sum_{j=1}^{N}\left|\frac{d_{i j}(t)}{d t}\right| \\
& \approx \frac{1}{N} \sum_{j=1}^{N}\left|\frac{d_{i j}\left(t_{2}\right)-d_{i j}\left(t_{1}\right)}{t_{2}-t_{1}}\right|
\end{aligned}
$$

Thus, either node $i$ stays away from $j$ or become closer, equation (3) can simplify into:

$$
\begin{aligned}
M_{i}(t) & =\frac{1}{N} \sum_{j=1}^{N}\left|\frac{d_{O B}\left(t_{2}\right)-d_{O A}\left(t_{1}\right)}{t_{2}-t_{1}}\right| \\
& =\frac{1}{N} \sum_{j=1}^{N}\left|\frac{d_{O B}\left(t_{2}\right)-d_{O A}\left(t_{1}\right)}{\Delta t}\right|
\end{aligned}
$$

In a MANET of $N$ nodes, the degree of node $i$, which is a good indicator of node density, can be easily denoted as:

$$
d_{i}=\left\{\begin{array}{cc}
1 & 0<d_{i j}<R \\
0 & \text { otherwise }
\end{array}\right.
$$

where $d_{i j}$ is the distance between node $i$ and $j, i=1,2, \ldots, N, j=1,2, \ldots, N$. The bigger $d_{i}$, the more centric of node $i$ is.

Thus, the relative node degree of node $i$ can be calculated as $D_{i}=\left|d_{i}-\sqrt{N}\right|$.

When choosing cluster-heads, our clustering algorithm take the mobility factors and node's relative degree into account, and make a reasonable compromise based on the actual needs and operation environment. Therefore, we use a combined weighted algorithm to select cluster-heads, which can improve overall performance of MANETs. Each node is assigned a weight indicating whether the node is suitable to act as a cluster-head. So, the cost metric (represented as $C$ for simplicity) of node $i$ can be expressed as:

$$
C_{i}=w_{1} \frac{1}{M_{i}(t)}+w_{2} D_{i}
$$

Where $i=1,2, \ldots, N, w_{1}$ and $w_{2}$ are the weight factor of the parameter $M_{i}(t)$ and $D_{i}$ respectively, and the more important parameters, the greater its weight factor is. $w_{1}$ and $w_{2}$ can be pre-established or adjusted adaptively according to real-life network, but $w_{1}+w_{2}=1$. Here, we set the initial value of $w_{1}$ and $w_{2}$ to $1 / 2$ and $1 / 2$ respectively, and adjusted them adaptively according to real-life network.

\subsection{Clustering Algorithm}

Clustering is a mechanism to dynamically group nodes in MANETs into logically separating or overlapping entities, which is called clusters. In our scheme, there are five possible states for nodes: NULL, cluster-head, cluster-member, cluster-gateway, and cluster-guest.

In this paper, we usually consider one-hop clusters, besides the only scenario, namely, when the cluster-guest appears, in this case, the guest node is two-hop away from the cluster-head. All the nodes in such a cluster are within the range of the cluster-head, but not necessarily within range of each other.

1) Cluster Initialization

Prior to the cluster initialization, all nodes are in the state of NULL. Once started, each node in the network broadcasts a HELLO message to have knowledge of its member nodes, which can be used to calculate its cost metric. Then, each node broadcasts a CH_ELECT message piggybacking the cost 
metric, calculated by the algorithm in section 2.1, to its neighbor. Upon receiving, it will compare the cost metric with itself, and the bigger will be elected as a cluster-head. Finally, the cluster-head will broadcast the elected result message called CH_CLAIM (cluster-head claim) to its one-hop neighbors. Upon receiving, the neighbors will send a REQ (route request) message to the cluster-head, and cluster-head will send a REP (route reply) back if agree.

After the above process, some clusters will have been formed. But when a cluster-member receives more than one REP message, this denotes that the node lies in separate clusters but within transmission range of one another, therefore it will be elected as a gateway between these clusters.

2) Cluster Maintenance

Once the initial clustering phrase takes place, cluster-heads and cluster-members must exchange message to maintain the relationship periodically. Namely, the cluster-head periodically broadcasts CH_CLAIM(clusterID) messages to its neighboring nodes. And the cluster-members of the attached cluster broadcasts cluster-memeber(nodeID, clusterIDs) messages back to the cluster-head periodically, where the nodeID is the identifier of the broadcasting node, and clusterID is the list of clusters of which the node is a member. When topology changes, we can deal with it according to the three cases as follow.

(1) delete or add nodes

A cluster-member would dissociate from the attached cluster, if it does not hear periodic broadcast from its cluster-head. Likewise, the cluster-head will remove the cluster-member from its list of members, if it does not receive the periodically cluster-member broadcasts.

When a node, including new coming or dissociated from other clusters, wants to join in a cluster, it should sends REQ to a cluster-head, and the cluster-head will sends a REP message back only if the requesting node is allowed to join in.

Note that when a node moves out of its cluster-head' transmission range but still has a link to another cluster-member belonging to any cluster-head, it will become a cluster-guest to avoid a new initial clustering formation takes place, despite the cluster-guest's cost metric is bigger or not. In this way, it can reduce the cluster-head change rate, and the ripple effects which caused by reclustering can be ignored. Hence the routing overhead will be decreased.

(2) replace the cluster-head position

Once a cluster-head leaves its own cluster or damaged, the node belonging to this cluster would return to the NULL state. Thus, they should be request to join in other clusters or establish a new cluster. Note that only when the nodes receive more than two consecutive REQ message from certain node, should they establish a new cluster.

(3) merge of different two clusters

When a cluster enters the transmission range of another cluster and the variance of cost metric of the two cluster-head is small, which denotes that the two clusters is worth to merge. If so, the cluster-head which has bigger metric will be reelected as the new cluster-head, but the similar must give up its cluster-head role to be a common member of the new cluster. Otherwise, it denote that the two clusters is just incidentally pass by each other in a short period and has no worthy to merge. In this way, it can reduce the probability of cluster overlapping.

\section{Clustering Routing Protocol}

In order to reduce the route overhead and utilize the network resources efficiently, the proposed routing protocol uses proactive protocol in inter-cluster and reactive protocol in intra-cluster communication.

\subsection{Intra-cluster Communication}

If the source and destination are in the same cluster, the data packet can be transmitted directly or relayed by cluster head. Namely, when the destination is in the range of source, they can communicate with each other directly or relayed by cluster-head. Otherwise, they must be exchange data through cluster-head. 


\subsection{Inter-cluster Communication}

If the source and destination are in the different clusters, the source must take the intra-cluster strategy. Namely, at the first, the source sends a REQ (request) message to its attached cluster-head, and then the cluster-head will broadcast this REQ to its adjacent cluster-head through gateway nodes, and the process will continued until the REQ arrives at the cluster which belongs to the destination node. Finally, the cluster-head including the destination sends a REP (responding) message back along the discovered path. Note that the cluster-head in the discovered path will transfer the REP along the local shortest route. Thus, the source will get the shortest route to the destination.

\subsection{Route Maintenance}

Due to the frequent topology detection, an efficient and effective method of route maintenance in response to underlying network topology change is imperative because without routes validity the performance of a routing scheme in a dynamic, mobile environment is affected, and even adversely.

When an existing link is failure (such as the node on the existing path moves out of its one-hop neighbor or exists from the network, or the receiving node on the existing path cannot receive message from sending node because of deterioration of the channel), the local repairing process would take place. Namely, an existing shorter path will replace the original route between the two nodes which link is broken. Meanwhile, a CLEAR message will be forwarded to the source node which originates the packets to notify the change.

Note that in order to ensure the validity and stability of route, the local repairing process can be took place, only when the inter-cluster routes invalidated. And this mechanism is very helpful to reduce the route reestablishing expenses and end-to-end delay.

\section{Performance Analysis}

All manuscripts must be in English, also the table and figure texts, otherwise we cannot publish your paper. Please keep a second copy of your manuscript in your office. When receiving the paper, we assume that the corresponding authors grant us the copyright to use the paper for the book or journal in question. Should authors use tables or figures from other Publications, they must ask the corresponding publishers to grant them the right to publish this material in their paper. In our simulation experiments, we implemented the clustering algorithm and clustering routing protocol in the simulator NS2. The simulation is done in $300 \times 300 \mathrm{Km}$ area, each node has a same transmission range of $10 \mathrm{Km}$, and the maximum moving speed of each is $40 \sim 80 \mathrm{~m} / \mathrm{s}$. The MAC layer used the TDMA (Time Division Multiple Access) protocol, with a channel bandwidth of 5 Mbps. Traffic sources are CBR (constant bit rate), with the rate of 10 packets per second and 512 bytes per packet. The physical layer uses the free space model (Physical layer character takes DSSS), and takes the actual aeronautical communication parameters into account, such as the emission power is $125 \mathrm{~W}$ and receiver sensitivity is $-24.67 \mathrm{dBm}$. The intra-cluster communication takes the OLSR (Optimized Link State Routing) proactive protocol, but inter-cluster takes the AODV (Ad-Hoc On-Demand Distance Vector) reactive protocol. The simulation time is set to $60 \mathrm{~min}$.

\subsection{Stable Clustering Algorithm Analysis}

In this section, we use four evaluation criteria to evaluate the performance of the clustering algorithm: the number of clusters with respect to nodes' number, nodes' switch times between clusters with respect to nodes' number, average cluster-head holding time with respect to speed and average cluster-head holding time with respect to transmission range. And the proposed clustering algorithm is simulated against other three existing clustering algorithm, namely, DDVC, DLDC and MPBC. The nodes linearly move in a single direction, where their direction is initially randomly chosen. 


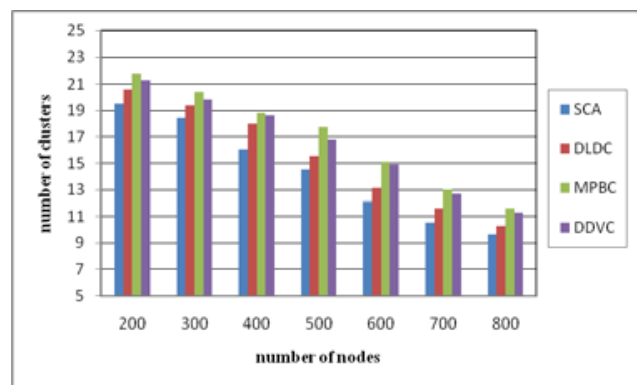

Fig. 1 clusters' number

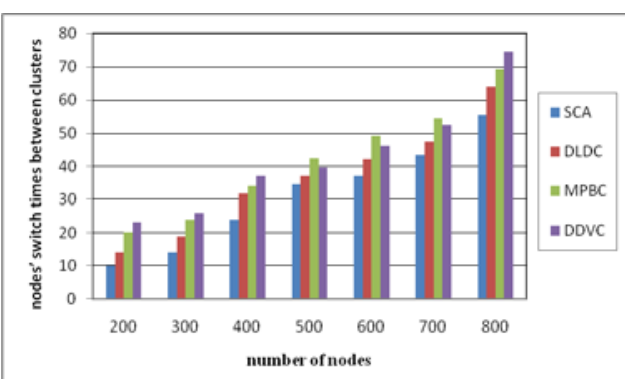

Fig. 2 nodes' switch times between clusters

Fig. 1 depicts the influence of the nodes' number to the number of clusters, which indicates the overhead of the network at the cluster-head level. From Fig. 1, it can be seen that the number of clusters formed using SCA surpass the number of clusters formed using the DDVC, DLDC and MPBC schemes. The SCA scheme outperforms the others as it takes the mobility factors and node's relative degree into account.

With the increase of nodes' density, this will lead to frequent topology changes and hence more establishing and disconnecting of the links. Fig. 2 depicts the results. From Fig. 2, it can be seen that the SCA scheme outperforms the others as it creates less clusters (hence, more cluster-members when the nodes' number is fixed). And because the proposed of the role of cluster-guest in SCA scheme, which avoids a new cluster formation process, took place, therefore the nodes' switch times between clusters is decreased. This indicates that the SCA algorithm performs more stability when the density of nodes increases.
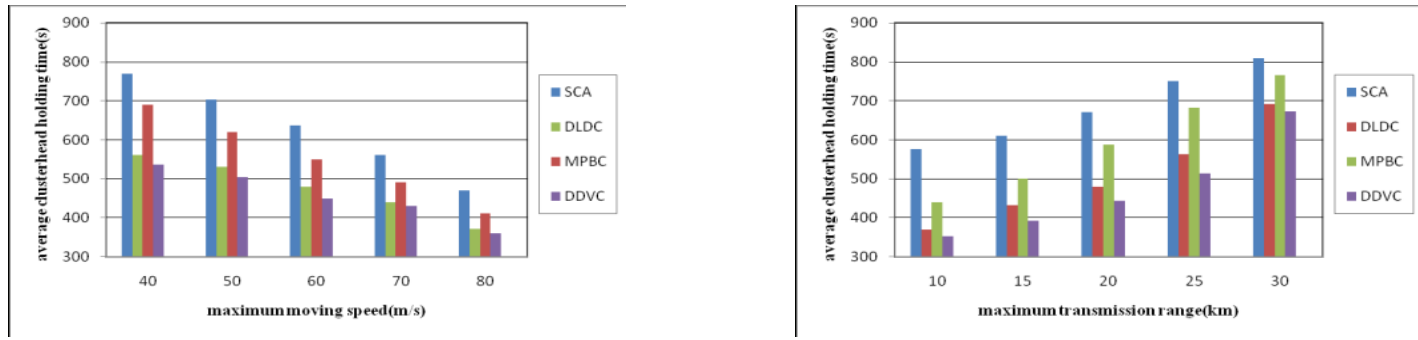

Fig. 3 average cluster-head holding time

Fig. 3(a) depicts the average cluster-head holding time with respect to nodes' maximum moving speed. From Fig. 3(a), it can be seen that with the speed increasing, the average cluster-head holding time of all the schemes is decreased. This is because of the network topology is changing frequently, when nodes' speed growing up, which leads to more re-clustering. As a result of it, the cluster-head holding time is decreased. But the SCA scheme outperforms the others as it takes the mobility factors and node's relative degree into account. So, its cluster-head holding time is longer than others.

Fig. 3(b) depicts the average cluster-head holding time with respect to nodes' maximum transmission range. From Fig. 3(b), it can be seen that the SCA scheme' cluster-head holding time is longer than others. And the gap between all the schemes is small, as when the transmission range is expanding, the influence of node's mobility to the link is debasing.

\subsection{Routing Protocol Analysis}

In this section, we use three evaluation criteria to evaluate the performance of our routing protocol: average end-to-end delay, normalized routing overhead, and packet acceptance ratio.

Average end-to-end delay includes route finding, transmission time in MAC layer and physical channel, which indicates the connectivity and efficiency of the network. Normalized routing overhead is the ratio of the total number of control message transmissions (the forwarding of a control message at each hop is counted as one control transmission) to the total number of data packet received, which indicates the efficiency of the routing protocol. Packet acceptance ratio is the ratio of the received data packet number at source to the sent data packet number at destination. This indicates the reliability of the network.

This section will compare the SCA-R protocol with other three protocols, i.e., MPBC-R, DLDC-R and DVDC-R. 
It is obviously that the more nodes will lead to bigger average end-to-end delay. This is because the more nodes participate in the network communication, the more bandwidth will be consumed, and hence more congestion will be took place, so the average end-to-end delay will increase substantially. Fig. 4 demonstrates the average end-to-end delay with respect to nodes' number of all the schemes. From Fig. 4, it can be seen that the SCA-R outperforms other as it takes the mobility factors and node's relative degree into account, and hence more stable clusters it will be established. Thus, it will cost little time to communicate with each other.

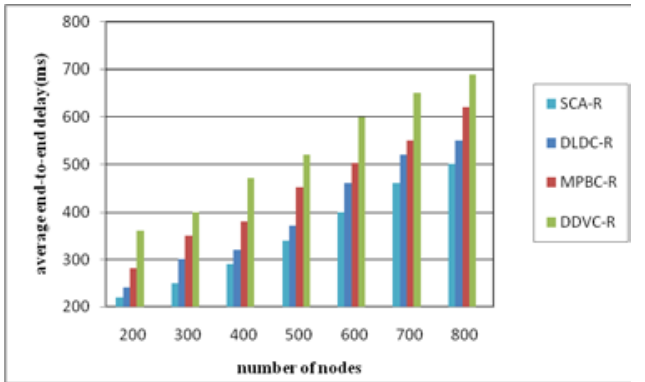

Fig. 4 average end-to-end delay

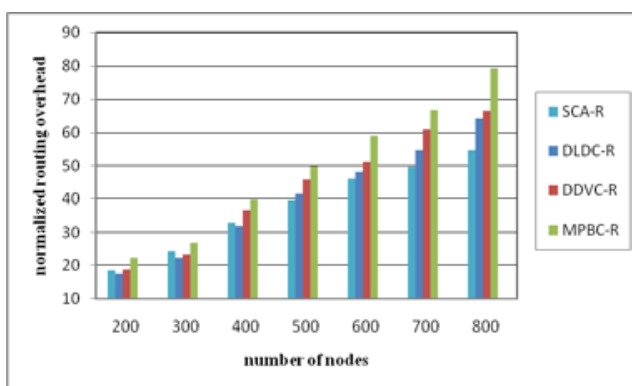

Fig. 5 normalized routing overhead

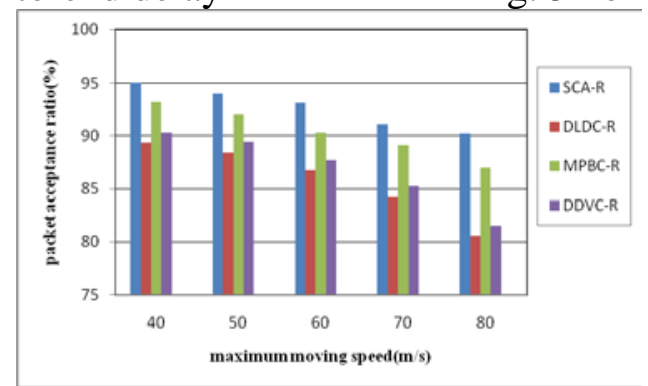

Fig. 6 packet acceptance ratio

Network routing overhead mainly comes from the control message exchanges, e.g., REQ, REP and CLEAR packet, in the routing re-establishing and maintenance processes. Fig. 5 demonstrates the normalized routing overhead with respect to node's number of all the schemes. From Fig. 5, it can be seen that the SCA-R protocol outperforms other as it uses proactive protocol in inter-cluster and reactive protocol in intra-cluster communication, and hence restricts the flooding area effectively. And the role of cluster-guest decreases the new clustering process takes place, so little routing overhead will be obtained.

Fig. 6 demonstrates the packet acceptance ratio with respect to maximum moving speed. From Fig. 6 , it can be seen that as the speed growing up, the packet acceptance ratio of all the schemes is going down. It is due to the bigger speed lead to more frequent topology changes, and hence more broken links or unreachable downstream nodes appear. So, more data packets will be discarding. The SCA-R routing protocol outperforms others as it established more stable clusters. Therefore, the higher packet acceptance ratio it will be.

\section{Conclusion}

Based on the analysis of existing clustering routing protocols and clustering algorithms, a stable clustering algorithm based on the cost metric and a corresponding hierarchical routing protocol were proposed. Simulation results show that the proposed stable clustering algorithm and hierarchical routing protocol provide superior performance with several advantages over existing clustering algorithm and routing protocol respectively.

\section{Acknowledgments}

The communicated author is Jinke Huang, and his Email is 86297609@qq.com. 


\section{References}

[1] Conti M, Giordano S. Mobile ad hoc networking: milestones, challenges, and new research directions[J]. IEEE Communications Magazine, 2014, 52(1): 85 96.

[2] Swidan A, Khattab S, Abouelseoud Y and Elkamchouchi H. A secure geographical routing protocol for highly-dynamic aeronautical networks[C]// MILCOM 2015 - 2015 IEEE Military Communications Conference, Tampa, FL: IEEE Press, 2015. $26 \sim 28$.

[3] Gnanambigai J, Rengarajan N and Navaladi N. A clustering based hybrid routing protocol for enhancing network lifetime of Wireless Sensor Network[C]// 2014 2nd International Conference on Devices, Circuits and Systems (ICDCS), Combiatore: IEEE Press, 2014. 6 8.

[4] Cherine Fathy, Mahmoud T. El-Hadidi and Mohamad Abou El-Nasr. Fuzzy-based adaptive cross layer routing protocol for mobile ad hoc networks[C]// 30th IEEE International Performance Computing and Communications Conference, Orlando, FL: IEEE Press, 2011. 1 10.

[5] VanDung Nguyen, Oanh Tran Thi Kim, Duc Ngoc Minh Dang, Sung Soo Kim and Choong Seon Hong. Application of the lowest-ID algorithm in cluster-based TDMA system for VANETs[C]// 2015 International Conference on Information Networking (ICOIN), Cambodia: IEEE Press, 2015. 25 30.

[6] Zhang Y, Ng J M . A Distributed Group Mobility Adaptive Clustering Algorithm for Mobile Ad Hoc Networks[C]// 2008 IEEE International Conference on Communications, Beijing: IEEE Press, 2008. 3161 3165.

[7] ShengYi J, Xia L. Lowest-ID with adaptive ID reassignment: a novel mobile ad-hoc networks clustering algorithm[C]// Sixth International Conference on Fuzzy Systems and Knowledge Discovery, Tianjin: IEEE Press, 2009. 366 370.

[8] Ehssan Sakhaee and Abbas Jamalipour. Stable clustering and communications in pseudolinear highly mobile ad hoc networks[J], IEEE Transactions on Vehicular Technology, 2008, 57(6): 3769 3777.

[9] Minming N, Zhangdui Z, and Dongmei Z. MPBC: a mobility prediction-based clustering scheme for ad hoc networks[J], IEEE Transactions on Vehicular Technology, 2011, 60(9): 4549 4559.

[10] Tinku Rasheed, Khaldoun Al Agha, Usman Javaid and Laurent Reynaud. Cluster-Quality Based Hybrid Routing for Large Scale Mobile Multi-Hop Networks[C]// 2007 IEEE Wireless Communications and Networking Conference, Kowloon: IEEE Press, 2007. 3052 3057.

[21] Yu T, Linhua M, Hong T and Song Z. A Signal Characteristic Based Cluster Scheme for Aeronautical Ad Hoc Networks[J]. IEICE Transaction on Communications, 2014, E97-B(2): $441 \sim 449$.

[12] Xiaoyan H, Gerla M, Yunjung Y, Kaixin X and Taek Jin Kwon. Scalable ad hoc routing in large, dense wireless networks using clustering and landmarks[J]. IEEE International Conference on Communications, 2002, 5(5): 3179 3185. 